\title{
HEMES FÉRRICOS PENTACOORDENADOS E HEXACOORDENADOS DOS MONÔMEROS $d$ NATIVO E RECONSTITUIIDO DA HEMOGLOBINA EXTRACELULAR DE Glossoscolex paulistus: ESTUDOS ESPECTROSCÓPICOS EM MEIO ÁCIDO
}

\author{
Julio C. Ribelatto*, Alessandra L. Poli, Leonardo M. Moreira e Hidetake Imasato \\ Instituto de Química de São Carlos, Universidade de São Paulo, CP 780, 13560-970 São Carlos - SP
}

Recebido em 24/8/04; aceito em 4/1/05; publicado na web em 13/4/05

\begin{abstract}
PENTACOORDINATE AND HEXACOORDINATE FERRIC HEMES FROM THE NATIVE AND RECONSTITUTED $d$ MONOMERS OF Glossoscolex paulistus EXTRACELLULAR HEMOGLOBIN: SPECTROSCOPIC STUDIES IN ACID MEDIUM. UV-Vis and fluorescence spectroscopic studies of the native and reconstituted $d$ monomers of Glossoscolex paulistus were performed in acid medium. The coexistence of distinct species shows the complexity of the equilibria. Besides the hexacoordinate low spin hemichrome, with bands at 535 and $565 \mathrm{~nm}$, a pentacoordinate high spin hemichrome is identified by the blue-shifted low intensity Soret band (371 nm) and the LMCT band $(643 \mathrm{~nm})$. The pentacoordinate hemichrome must be related to the partial unfolding of the polypeptide.
\end{abstract}

Keywords: extracellular hemoglobin; hexacoordinate; pentacoordinate.

\section{INTRODUÇÃO}

A hemoglobina $(\mathrm{Hb})$ extracelular gigante de Glossoscolex paulistus é uma hemoproteína oligomérica ${ }^{1}$ com uma massa molecular de 3,1 $\mathrm{MDa}^{2}$. Pertencente à mesma classe do anelídeo Lumbricus terrestris, que tem sido intensamente investigado ${ }^{3-9}$, essa hemoglobina ainda permanece intrigante sob vários aspectos da sua relação estrutura/atividadeSua estrutura apresenta 144 globinas e 36 cadeias polipeptídicas sem o grupo prostético heme que são cruciais para sua integridade estrutural (cadeias "linkers") ${ }^{10}$, sendo que o arranjo da hemoglobina íntegra se dispõe espacialmente como 2 discos hexagonais sobrepostos, com as subunidades estabilizadoras, "linkers" na parte central". As 144 globinas estão organizadas como 12 dodecâmeros, sendo que cada dodecâmero consiste em um conjunto de 3 tetrâmeros. Cada tetrâmero, por sua vez, é constituído por 3 trímeros (cadeias $a, b, c$ unidas covalentemente através de ligações dissulfeto) e 1 cadeia monomérica $d^{6,11}$.

Tendo-se em vista a menor complexidade do estudo biofísico de uma única subunidade ao invés da proteína íntegra propriamente dita, o monômero $d$, previamente modelado por Cabral e colaboradores $^{12}$, foi isolado visando a caracterização das espécies presentes em equilíbrio químico, em meio ácido. De fato, a complexidade do equilíbrio de hemoproteínas vem motivando estudos em função da coexistência de várias espécies em cada valor de $\mathrm{pH}^{13-18}$, sendo que estas podem diferir em relação ao estado de spin e aos ligantes axiais propriamente considerados. Neste particular, pode haver espécies com números de coordenação diferenciados, ou seja, é possível a coexistência de formas hexacoordenadas, pentacoordenadas ${ }^{13,18} \mathrm{e}$, até mesmo, tetracoordenadas ${ }^{19,20}$.

Vários trabalhos vêm enfatizando a relação entre a formação de espécies pentacoordenadas e um processo inicial de desenovelamento da cadeia polipeptídica, o que aumenta, ainda mais, a relevância da caracterização destas espécies, devido a suas possíveis implicações com fenômenos de desnaturação ${ }^{19,20}$.

Por outro lado, sabe-se que há reconstituições de hemoproteínas que implicam na perturbação da estrutura terciária ${ }^{21}$, assim como

*e-mail: ribelatto@iqsc.usp.br outras que não afetam significativamente a mesma ${ }^{22}$; da mesma forma, há reconstituições que aumentam a afinidade do heme pelo sexto ligante axial ${ }^{23}$ e outras, que diminuem esta constante de estabilidade $^{24}$. Por conseguinte, o processo de reconstituição poderia afetar as propriedades do monômero de diversas formas em comparação às propriedades do nativo. O estudo do monômero reconstituído, no presente trabalho, visa elucidar as possíveis mudanças que poderiam ocorrer na primeira esfera de coordenação do centro férrico em função do $\mathrm{pH}$, refletindo, inclusive, nos valores de $\mathrm{pK}$ observados.

De fato, têm sido desenvolvidas reconstituições de hemoglobinas buscando a elucidação das orientações possíveis e preferenciais do heme ${ }^{25}$, a análise da influência das propriedades do heme sobre a cadeia polipeptídica e a avaliação da sensibilidade da estrutura terciária às perturbações impostas ao heme ${ }^{21,22}$, além de trabalhos sobre eventuais modificações na afinidade pelo sexto ligante axial, com destaque para o principal ligante deste sistema no meio biológico, isto é, o oxigênio molecular ${ }^{23,24}$.

Portanto, o presente estudo aborda a complexidade dos equilíbrios desta subunidade monomérica em meio ácido, assim como os possíveis processos envolvidos na primeira esfera de coordenação do centro férrico.

\section{PARTE EXPERIMENTAL}

\section{Obtenção e purificação: oxi-hemoglobina íntegra, meta-} hemoglobina e monômero

A hemolinfa foi extraída com auxílio de pipetas de Pauster em presença de anti-coagulante (citrato de sódio $0,1 \mathrm{mmol} \mathrm{L}^{-1}$ ) e armazenada em banho gelado durante a coleta. A primeira etapa da purificação foi realizada através de centrifugação da hemolinfa a $500 \mathrm{rpm}$ por $10 \mathrm{~min}$ a $4{ }^{\circ} \mathrm{C}$, para precipitação de impurezas. Em seguida, realizou-se uma diálise contra tampão TRIS-HCl $100 \mathrm{mmol} \mathrm{L}^{-1}, \mathrm{pH} 7,0 \mathrm{com} 0,1 \mathrm{mmol} \mathrm{L}{ }^{-1}$ EDTA. Após a diálise, efetuou-se a ultracentrifugação da proteína a $4^{\circ} \mathrm{C}$ por $6 \mathrm{~h}$ a 35000 rpm e, ato contínuo, promoveu-se nova suspensão em tampão TRIS$\mathrm{HCl} 100 \mathrm{mmol} \mathrm{L}^{-1}$, pH 7,0 com EDTA $0,1 \mathrm{mmol} \mathrm{L} \mathrm{L}^{-1}$. A purificação foi completada por cromatografia de exclusão em coluna de gel 
Sephadex G-200 equilibrada com tampão TRIS-HCl $100 \mathrm{mmol} \mathrm{L}^{-1}$, pH 7,0, contendo EDTA $0,1 \mathrm{mmol} \mathrm{L}^{-1}$. Como o eluato coletado no volume de exclusão contém a oxiHb íntegra, este é estocado a $4{ }^{\circ} \mathrm{C}$ para uso posterior ${ }^{26}$. A fração oxi-monômero foi separada filtrando-se a oxiHb íntegra estabilizada em pH 9,0 em uma coluna de gel de Sephadex G-200². A fração meta-monômero foi separada filtrando-se a meta-hemoglobina estabilizada em pH 9,0 em uma coluna de gel de Sephadex G-200. A meta-hemoglobina foi obtida através de uma reação de oxidação da oxiHb íntegra com ferricianeto de potássio na razão molar de 3:1 em relação à concentração de heme $e^{6,27}$.

\section{Obtenção do apomonômero}

O apomonômero foi isolado a partir da forma oxi-monômero, sendo que o grupo heme foi extraído com metiletilcetona após ajustar-se o $\mathrm{pH}$ para 2,8, consoante metodologia estabelecida por Teale ${ }^{28}$.

\section{Reconstituição do monômero}

A solução de hemina de mioglobina equina foi preparada empregando-se um volume mínimo de $\mathrm{NaOH} 0,2 \mathrm{~mol} \mathrm{~L}^{-1}$, lograndose um estoque de $0,153 \mu \mathrm{mol} \mathrm{L^{-1 }}$. A reconstituição foi realizada pela adição de alíquotas de uma solução de apomonômero a uma solução de hemina $\left(1 \mu \mathrm{mol} \mathrm{L} \mathrm{L}^{-1}\right)$ obtida pela diluição da solução estoque em uma solução de TRIS-HCl $20 \mathrm{mmol} \mathrm{L}^{-1}$ contendo $1 \mathrm{mmol} \mathrm{L}^{-1}$ de EDTA, obtendo-se um $\mathrm{pH}$ de trabalho igual a 7,0.

\section{Espectroscopia de absorção na região do ultravioleta-visível}

Os espectros de absorção foram medidos entre 250-700 nm, em aparelho Shimadzu UV-1601 PC à temperatura ambiente. Os espectros foram armazenados com o objetivo de serem posteriormente analisados, empregando-se o programa CCA ("convex constraint analysis") ${ }^{29}$. O programa CCA permite calcular os espectros das espécies químicas que coexistem em solução (espectros que foram denominados de espectros bases) através da análise do conjunto dos espectros experimentais (funções iniciais). Um espectro de absorção em uma determinada condição experimental pode ser representado pela função $A(\lambda)$, Equação 1 :

$A(\lambda)=\sum_{i=1}^{P} a_{i} A_{i}(\lambda)$

onde $a_{i}$ é o coeficiente que determina a proporção do constituinte $i$; $A_{i}(\lambda)$ são os espectros das soluções das espécies químicas $i$, constituindo-se nos espectros bases; $P$ é o número total de espécies químicas em solução. As restrições impostas pelo programa são:

1) $\sum_{i=1}^{P} a_{i}=1$;2) $a_{i} \geq 0$;3) $A_{i}$ 's são linearmente independentes

Em resumo, os espectros $-A_{i}(\lambda)$ - e as proporções - $a_{i}$ - são determinadas através de avaliação estatística "convex constraint".

\section{Medidas de fluorescência}

Para as medidas da emissão de fluorescência dos monômeros $d$ nativo e reconstituído da $\mathrm{Hb}$ de G. paulistus nas formas meta em pH 7,0 foi utilizado um espectrofluorímetro HITACHI F-4500, usando uma cubeta de quartzo com as quatro faces polidas e caminho ótico de $1 \mathrm{~cm}$. Os valores de $\mathrm{pH}$ foram ajustados utilizando-se alíquotas de uma solução estoque de $\mathrm{HCl}$, variando-se o $\mathrm{pH}$ a cada 0,5 unidade na faixa de 7,0 a 3,0 . O comprimento de onda de exci- tação foi de $295 \mathrm{~nm}$ e os espectros de emissão foram obtidos entre 305 a $450 \mathrm{~nm}$. As amostras foram preparadas com absorbância em $295 \mathrm{~nm}\left(\mathrm{~A}_{295}\right) \leq 0,1$, com o objetivo de se evitar os efeitos de absorção interna. Todos os resultados foram normalizados para um valor de $\mathrm{A}_{295}=0,1$.

\section{RESULTADOS E DISCUSSÃO}

\section{Espectros de absorção na região do ultravioleta-visível em função do pH}

Os espectros de absorção dos monômeros $d$ nativo e reconstituído da $\mathrm{Hb}$ G. paulistus foram obtidos inicialmente em $\mathrm{pH}$ 7,0. O monômero nativo, por sua vez, foi obtido por dissociação alcalina, sendo necessário o ajuste do $\mathrm{pH}$ para 7,0. Esse processo cromatográfico em $\mathrm{pH}$ 9,0 proporciona a formação do chamado hemicromo (complexo bis-histidina constituindo uma configuração eletrônica de baixo spin ${ }^{15,17,30-32}$ ), ou seja, o grupo prostético heme, inicialmente na forma aquometa, torna-se um complexo bis-histidina, através da coordenação ao centro férrico do nitrogênio do imidazol proveniente da histidina distal.

Nas Figuras 1(a) e 2(a) são apresentados os espectros referentes aos monômeros $d$ nativo e reconstituído, respectivamente. Nos dois conjuntos de espectros, observa-se que em $\mathrm{pH} 7,0$, as bandas de Soret estão centradas em $413 \mathrm{~nm}$ e as bandas Q ( $\alpha$ e $\beta$ ) possuem um máximo em 565 e $535 \mathrm{~nm}$, sendo estas, características de hemicromo $0^{30,31,33-35}$.
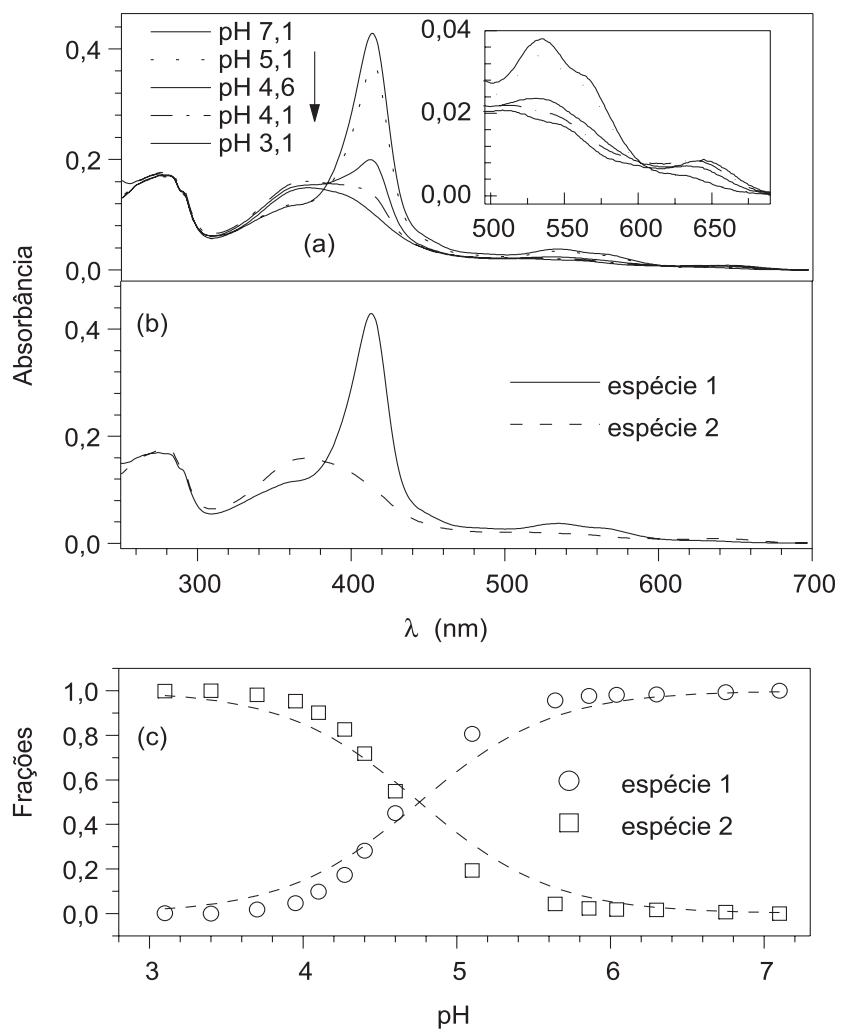

Figura 1. (a) Espectros eletrônicos do monômero d nativo da metaHb de Glossoscolex paulistus em função do $\mathrm{pH}$; (b) espécies predominantes (hemicromo - espécie 1 / espécie pentacoordenado - espécie 2) correspondentes aos componentes dos espectros experimentais em diferentes proporções em função do $\mathrm{pH}$; (c) porcentagem das frações de cada espécie em função do $\mathrm{pH}$. Ajustes (---) da fração de cada uma das espécies presentes no meio ácido. $O$ inserto em 1 (a) é uma ampliação das bandas $Q$ e de transferência de carga 

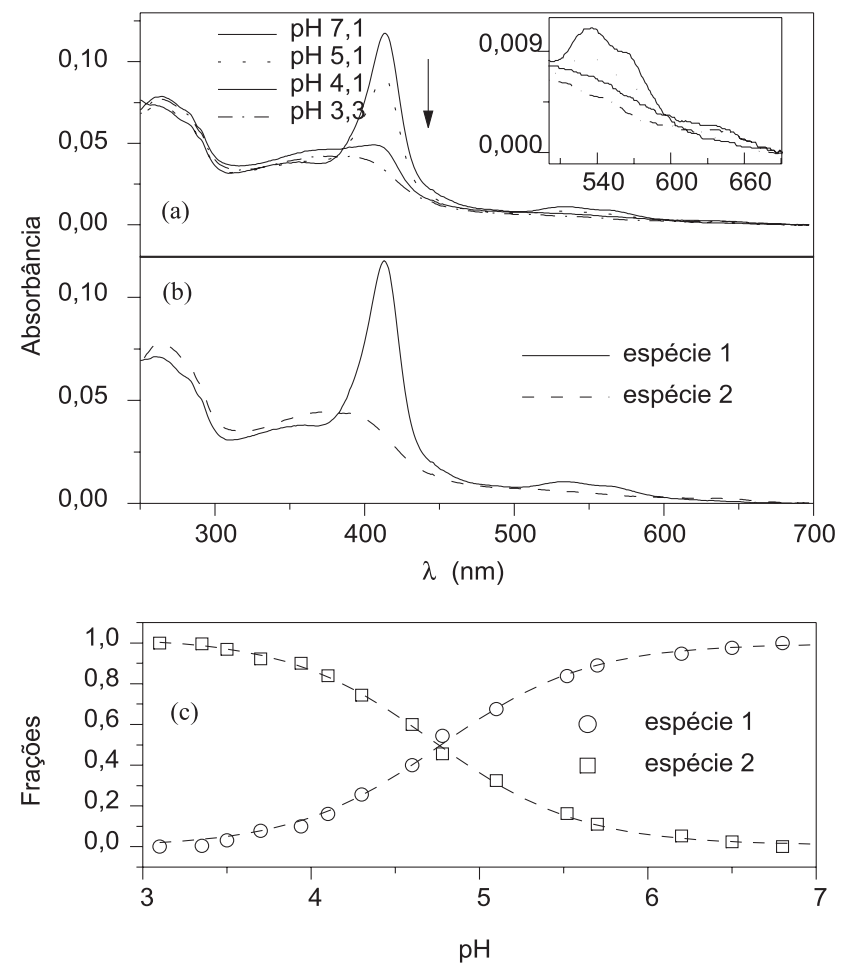

Figura 2. (a) Espectros eletrônicos do monômero d reconstituído da metaHb de Glossoscolex paulistus em função do $\mathrm{pH} ;(b)$ espécies predominantes (hemicromo - espécie $1 /$ pentacoordenado - espécie 2) correspondentes aos componentes dos espectros experimentais em diferentes proporções em função do $\mathrm{pH} ;$ (c) porcentagem das frações de cada espécie em função do pH. Ajustes (---) da fração de cada uma das espécies presentes no meio ácido. $O$ inserto em 2 (a) é uma ampliação das bandas $Q$ e de transferência de carga

Com a acidificação, observa-se tanto uma diminuição da intensidade como um deslocamento hipsocrômico da banda de Soret para $371 \mathrm{~nm}$, juntamente com um deslocamento batocrômico da banda de transferência de carga do ligante para o metal (TCLM) para $643 \mathrm{~nm}$.

Após a análise espectral utilizando o algoritmo $\mathrm{CCA}^{23}$, verificou-se a presença dos espectros nas Figuras $1 \mathrm{~b}$ e $2 \mathrm{~b}$ para os monômeros $d$ nativo e reconstituído, respectivamente. Nestas análises, a ocorrência de duas espécies químicas predominantes em solução foi assumida: hemicromo (configuração eletrônica de spin baixo, espécie 1), que prevalece em meio neutro, e a formação de uma espécie pentacoordenada (configuração eletrônica de spin alto, espécie 2).

Nas Figuras 1(c) e 2(c) são apresentadas as frações de cada espécie em função do $\mathrm{pH}$. O valor de $\mathrm{pK}$ encontrado através do ajuste dos dados foi de 4,7 , tanto para a forma nativa quanto para a reconstituída.

Vale lembrar, entretanto, que a presença da espécie aquometa deve ser considerada mínima, porém não inexistente, pois a formação de hemicromo em função da separação cromatográfica em $\mathrm{pH}$ 9,0 não é total, ou seja, uma fração da hemoglobina permanece aquometa, o que pode ser identificado pela ocorrência de uma banda característica dessa espécie em $630 \mathrm{~nm}^{31,33,36}$ entre os valores de pH 7,0 e 5,0, denotando a complexidade do equilíbrio químico em questão.

A formação de uma banda relativamente larga em $371 \mathrm{~nm}$ foi atribuída a uma espécie pentacoordenada ${ }^{19,37-39}$. De fato, vários autores sugerem que, partindo-se de um heme hexacoordenado, a for- mação de um heme pentacoordenado implica em uma diminuição significativa da absortividade molar e um deslocamento hipsocrômico da banda de Soret, sendo que a grandeza deste deslocamento é peculiar para cada hemoglobina ${ }^{37,40-46}$. Entretanto, o significativo deslocamento para $371 \mathrm{~nm}$, encontrado no presente caso, indica não somente a formação de uma espécie pentacoordenada, mas demonstra que esse processo ocorre simultaneamente a um desenovelamento parcial da cadeia polipeptídica, isto é, a formação da espécie pentacoordenado estaria intrinsicamente relacionada a um processo inicial de desnaturação ${ }^{41-43}$.

Consoante proposta estabelecida por Sage e colaboradores ${ }^{19}$, é possível que a formação da espécie pentacoordenada no meio ácido esteja relacionada à protonação das histidinas que, inicialmente em meio neutro, estariam menos acessíveis ao solvente. Portanto, dois fenômenos concomitantes devem estar ocorrendo: o desenovelamento parcial da cadeia polipeptídica e a protonação das histidinas. Deste modo, ocorreria uma competição entre os prótons do meio ácido e o centro férrico pelo par de elétrons do nitrogênio do imidazol da histidina.

Alguns trabalhos ${ }^{43,47}$ estabelecem que, além da banda de Soret deslocada para o azul, uma banda de transferência de carga em 645 $\mathrm{nm}$ é característica de sistemas pentacoordenados ${ }^{43,47}$. Outros trabalhos, como o de Ikeda-Saito e colaboradores ${ }^{37}$, indicam o comprimento de onda $641 \mathrm{~nm}$, assim como Sage e colaboradores ${ }^{19}$ atribuem $640 \mathrm{~nm}$ para a banda característica desta espécie. No presente trabalho, tanto no monômero nativo como no reconstituído (Figuras 1 b e 2b), a referida banda ocorre em $643 \mathrm{~nm}$, indicando, uma vez mais, que a espécie pentacoordenada está presente em concentração representativa nas condições mais ácidas.

Com relação à caracterização do quinto ligante da espécie pentacoordenada, as informações da literatura são controversas. Alguns grupos propõem que, partindo-se de uma espécie aquometa, a quebra de ligação ocorreria entre o centro férrico e a histidina proximal ${ }^{19,20,38}$. Todavia, para o monômero $d$ isolado da hemoglobina de Glossoscolex paulistus, a espécie hemicromo é majoritária em $\mathrm{pH}$ 7,0 , ou seja, independentemente de qual ligação fosse quebrada, o centro de ferro pentacoordenado apresentaria como quinto ligante uma histidina, pois a espécie predominante em meio neutro é um complexo bis-histidina. Neste caso, o resíduo de aminoácido que permaneceria coordenado ao centro férrico deveria ser a histidina proximal, em função de sua ligação com o centro de coordenação ser menos tensionada do que a ligação envolvendo a histidina distal.

De qualquer maneira, Boffi e colaboradores ${ }^{48}$ têm estudado compostos porfirínicos pentacoordenados e justificado a estabilidade deste estado de coordenação, que é encontrado em várias hemoproteínas, em função da habilidade $\sigma$-doadora do quinto ligante. Assim sendo, ligantes que sejam bons $\sigma$-doadores, como por ex. água, imidazol e imidazolato, poderiam desestabilizar ligantes igualmente $\sigma$-doadores em sua posição trans, isto é, na sexta posição de coordenação $0^{39,44,49-51}$. Por outro lado, Bertini e colaboradores ${ }^{52}$ asseveram que a saída do centro férrico do plano porfirínico contribui estericamente para a estabilidade do pentacoordenado. Portanto, a influência trans labilizadora do quinto ligante e o impedimento estérico desta conformação atuariam sinergicamente dificultando a ligação de um sexto ligante, isto é, estabilizando a espécie pentacoordenada.

Ademais, há de se considerar que os deslocamentos da banda de transferência de carga estão intrinsicamente relacionados a uma saída pronunciada do centro férrico do plano porfirínico em direção ao quinto ligante. Esta saída do centro de coordenação é característica de hemes alto-spin, mais notadamente pentacoordenados ${ }^{37,44,53-56}$, e este fenômeno afetaria significativamente a superposição dos orbitais moleculares envolvidos nas ligações $\sigma$ e 
$\pi$ entre a porfirina e o centro férrico, o que, por conseqüência, alteraria o desdobramento dos orbitais envolvidos nas respectivas transições eletrônicas, implicando em modificações nos comprimentos de onda das bandas em questão.

Há de se adir, entretanto, que apesar da tendência de formação de uma espécie pentacoordenada conforme se acidifica o meio, existe uma coexistência de espécies diferenciadas em praticamente todos os valores de $\mathrm{pH}$, o que evidencia a complexidade dos equilíbrios do monômero $d$.

\section{Emissão de fluorescência em função do pH}

Efetuou-se um estudo sobre as propriedades de fluorescência dos monômeros $d$ nativo e reconstituído da $\mathrm{Hb}$ de G. paulistus, a partir de $\mathrm{pH}$ 7,0. Foram analisadas as emissões de fluorescência e a posição dos máximos de emissão. Nas Figuras 3 e 4, observa-se um aumento do rendimento quântico, a partir do $\mathrm{pH}$ 5,8 para o nativo e a partir de 5,5 para o reconstituído, sendo que os valores de pK avaliados foram respectivamente 4,9 e 4,7, similares, portanto, aos obtidos nos experimentos de espectroscopia de absorção eletrônica. Em relação aos comprimentos de onda de emissão, a acidificação leva a um deslocamento dos máximos de 326 para 329 $\mathrm{nm}$, corroborando a proposta de que o desenovelamento inferido pelas medidas de espectroscopia de absorção eletrônica é apenas parcial, não ocorrendo uma exposição significativa dos resíduos de triptofano ao solvente com a diminuição do $\mathrm{pH}$.

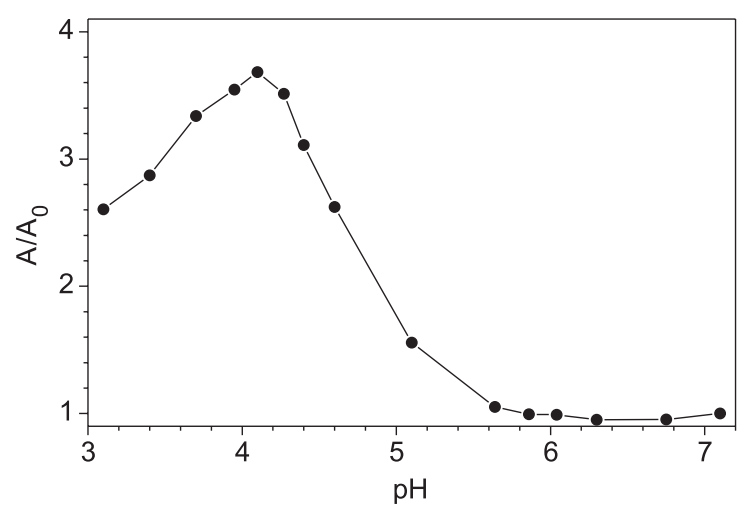

Figura 3. Áreas normalizadas dos espectros de emissão de fluorescência do monômero d nativo da metaHb de Glossoscolex paulistus, $\lambda_{\text {exc }}=295 \mathrm{~nm}$, $\lambda_{e m}=305-450 \mathrm{~nm}$, em função do $\mathrm{pH}$

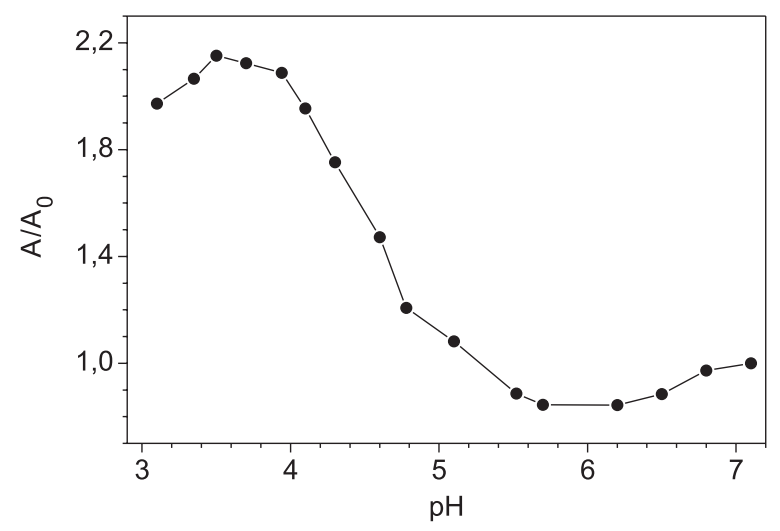

Figura 4. Áreas normalizadas dos espectros de emissão de fluorescência do monômero d reconstituído da metaHb de Glossoscolex paulistus, $\lambda_{\text {exc }}=$ $295 \mathrm{~nm}, \lambda_{\text {em }}=305-450 \mathrm{~nm}$, em função do $\mathrm{pH}$
Segundo o modelo do monômero $d$ proposto por Cabral e colaboradores $^{12}$, os resíduos de triptofano W33, W15, W129 encontram-se próximos à face distal do heme, inclusive com o resíduo W33 inserto dentro da cavidade hidrofóbica. No trabalho citado acima, os autores descreveram a interpretação do rendimento quântico de acordo com o mecanismo de Föster para a transferência de energia dos triptofanos para o heme. Na tentativa de se empregar esta abordagem, foi observada uma dificuldade significativa, que consiste no fato da integral de sobreposição entre o espectro de emissão do triptofano e o espectro de absorção do heme aumentar com a diminuição do $\mathrm{pH}$, opondo-se ao aumento do rendimento quântico observado com a acidificação. Contudo, é importante mencionar que ao se formar uma espécie pentacoordenada, com concomitante saída do centro férrico do plano dos nitrogênios pirrólicos em direção à histidina proximal ${ }^{37,44,48,55-57}$, a distância entre cada um dos triptofanos em questão e o centro férrico aumentaria. Sabendo-se que o grupo heme é o principal supressor da fluorescência de hemoglobinas e tendo-se em vista que, segundo o mecanismo de Föster, o aumento da distância entre o fluóroforo e o supressor intensifica o rendimento quântico do sistema devido à diminuição da transferência de energia, é possível que a formação da espécie pentacoordenada seja responsável pelo aumento da emissão de fluorescência observado para os monômeros $d$ nativo e reconstituído, em função do aumento da distância entre fluoróforos e supressor, cuja hipótese é reforçada pela coincidência dos valores de pK determinados pelas duas técnicas espectroscópicas utilizadas no presente trabalho.

Deste modo, o comportamento fluorescente dos monômeros $d$ nativo e reconstituído possivelmente está correlacionado à mudança conformacional decorrente da acidificação do meio, pois esse processo de desenovelamento protéico alteraria as distâncias e orientações dos triptofanos em relação ao heme.

Portanto, a formação da espécie pentacoordenada poderia estar relacionada com as emissões de fluorescência na faixa de $\mathrm{pH}$ estudada, tanto para o monômero nativo como para o reconstituído, haja visto a similaridade da evolução de rendimento quântico em ambos os sistemas. Outros estudos visando confirmar esta hipótese vêm sendo desenvolvidos em nosso grupo.

\section{CONCLUSÕES}

Através dos espectros de absorção, as bandas em 535 e 565 $\mathrm{nm}^{30,33,34}$, obtidas em $\mathrm{pH} 7,0$, tanto para o monômero $d$ nativo como para o reconstituído, indicaram que neste $\mathrm{pH}$ as subunidades monoméricas se encontravam, predominantemente, na forma de hemicromo. Todavia, a conversão de aquometa em hemicromo não é total, pois foi constatada uma banda característica da espécie aquometa em $630 \mathrm{~nm}^{31,33}$, que persiste no espectro mesmo após acidificação a pH 5,0.

Com uma maior acidificação, uma diminuição de intensidade e um deslocamento hipsocrômico foram observados para a banda de Soret, que são indicativos de um complexo pentacoordenado, assim como a banda de transferência de carga ligante para o metal (TCLM) em 643 nm. É provável que a formação desta espécie esteja intrinsicamente relacionada a um processo parcial de desnaturação.

Os estudos de fluorescência em meio ácido foram desenvolvidos partindo-se de $\mathrm{pH}$ 7,0 e foi demonstrado que aproximadamente em pH 5,7 se inicia um aumento do rendimento quântico do monômero $d$, o que deve estar relacionado à formação de uma espécie pentacoordenada.

Por conseguinte, no presente trabalho, as similaridades dos comportamentos espectroscópicos indicam que a ligação de um 
heme exógeno ocorre de forma específica na cadeia do apomonômero $d$ de G. paulistus, em função do processo de reconstituição.

\section{AGRADECIMENTOS}

À FAPESP pelo suporte técnico, à CAPES pela bolsa de mestrado proporcionada a J. C. Ribelatto e ao CNPq pela bolsa de doutorado concedida a L. M. Moreira.

\section{REFERÊNCIAS}

1. Costa, M. C. P.; Bonafé, C. F. S.; Meirelles, N. C.; Galembeck, F.; Braz. J. Med. Res. 1988, 21, 115.

2. Meirelles, N. C.; Oliveira, B.; Oliveira, A. R.; de Paula, E.; Marangoni, S.; Rennebeck, G. M.; Comp. Biochem. Physiol., Part A: Mol. Integr. Physiol. 1987, 88A, 377.

3. Martin, P. D.; Kuchumov, A. R.; Green, B. R.; Oliver, R. W. A.; Braswell, E. H.; Wall, J. S.; Vinogradov, S.N.; J. Mol. Biol. 1996, 255, 154.

4. Kuchumov, A. R.; Taveau, J. C.; Lamy, J. N.; Wall, J. S.; Weber, R. E.; Vinogradov, S. N.; J. Mol. Biol. 1998, 289, 1361.

5. Viana, E.; Silva, C. H. T. P.; Tabak, M.; Imasato, H.; Garrat, R. C.; Biochim. Biophys. Acta 1998, 1383, 130.

6. de Haas, F.; Kuchumov, A.; Taveau, J. C.; Boisset, N.; Vinogradov, S. N.; Lamy, J. N.; Biochemistry 1997, 36, 7330.

7. Mouche, F., Boisset, N.; Penczek, P. A.; J. Struct. Biol. 2001, 133, 176.

8. Krebs, A.; Lamy, J.; Vinogradov, S. N.; Zipper, P.; Biopolymers 1998, 45, 289 .

9. Zipper, P.; Krebs, A.; Durchschlag, P. Prog. Colloid Polym. Sci. 2002, 119, 141.

10. Royer Jr, W. E.; Strand, K.; van Hell, M.; Hendrickson, W. A.; Proc. Natl. Acad. Sci. U. S. A. 2000, 97, 7107.

11. Royer, W. E.; Knapp, J. E.; Strand, K.; Heaslet, H. A.; Trends Biochem. Sci. 2001, 26, 297.

12. Cabral, C. B.; Imasato, H.; Rosa, J. C.; Laure, H. J.; Silva, C. H. T. P.; Tabak, M.; Garrat, R. C.; Greene, L. J.; Biophys. Chem. 2002, 97, 139.

13. Dasgupta, S.; Rousseau, D. L.; Anni, H.; Yonetani, T.; J. Biol. Chem. 1989, 264,654 .

14. Levy, A.; Kuppusamy, P.; Rifkind, J. M.; Biochemisty 1990, 29, 9311.

15. Spagnuolo, C.; De Martino, F.; Boffi, A.; Rousseau, D. L.; Chiancone, E.; J. Biol. Chem. 1994, 269, 20441.

16. Wajnberg, E.; Bemski, G.; El-Jaick, J.; Alves, O. C.; Int. J. Biol. Macromol. 1996, 18, 231.

17. Svistunenko, D. A.; Sharpe, M. A.; Nicholis, P.; Wilson, M. T.; Cooper, C. E.; J. Magn. Reson. 2000, 142, 266.

18. Trent III, J. T.; Hvitved, A. N.; Hargrove, M. S.; Biochemistry 2001, 40, 6155.

19. Sage, J. T.; Morikis, D.; Champion, P. M.; Biochemistry 1991, 30, 1227.

20. Palaniappan, V.; Bocian, D. F.; Biochemistry 1994, 33, 14264.

21. Larsen, R. W.; Nunez, D. J.; Macleod, J.; Shiemke, A. K.; Musser, S. M.; Nguyen, H. H.; Ondrias, M. R.; Chan, S. I.; J. Inorg. Biochem. 1992, 48, 21.

22. Santucci, R.; Ascoli, F.; La Mar, G. N.; Pandey, R. K.; Smith, K. M.; Biochim. Biophys. Acta 1993, 1164, 133.

23. Cheng, Y.; Li, Y.; Li, R.; Lu, J.; Wang, K.; Chem.-Biol. Interact. 2000, 125, 191.

24. Bardgard, A. J.; Strand, I.; Nuutinen, M.; Jul, E.; Ole, B.; Comp. Biochem. Physiol., Part A: Mol. Integr. Physiol. 1997, 117, 367.

25. Yamamoto, Y.; Nakashima, T.; Kawano, E.; Chûjô, R.; Biochim. Biophys. Acta 1998, 1388, 349.
26. Imasato, H.; Tinto, M. H.; Perussi, J. R.; Tabak, M.; Comp. Biochem. Physiol., Part B: Biochem. Mol. Biol. 1995, 112, 217.

27. Zhu, H.; Ownby, D. W.; Rigs, C. K.; Nolasco, N. J.; Stoops, J. K.; Riggs, A F.; J. Biol. Chem. 1996, 271, 30007.

28. Teale, F. W. J.; Biochim. Biophys. Acta 1959, 35, 543.

29. Perczel, A.; Park, K.; Fasman, G. D.; Anal. Biochem. 1992, 203, 83.

30. Rachilewitz, E. A.; Peisach, J.; Blumberg, W. E.; J. Biol. Chem. 1971, 246, 3356.

31. Vitagliano, L.; Bonomi, G.; Riccio, A.; Prisco, G. D.; Smulevich, G.; Mazzarella, L.; Eur. J. Biochem. 2004, 271, 1651.

32. Sugawara, Y.; Kadono, E.; Suzuki, A.; Yukuta, Y.; Shibasaki, Y.; Nishimura, N.; Kameyama, Y.; Hirota, M.; Ishida, C.; Higuchi, N.; Haramoto, K.; Sakai, Y.;Soda, H.; Acta Physiol. Scand. 2003, 179, 49.

33. Antonini, E.; Rossi-Bernardi, L.; Chiancone, E.; Methods in Enzymology, $1^{\text {th }}$ ed.; Academic Press, 1981.

34. Riccio, A.; Vitagliano, L.; Prisco, G. D.; Zagari, A.; Mazzarella, L.; Acta Crystallogr., Sect. D: Biol. Crystallogr. 2001, 57, 1144.

35. Tsuruga, M.; Matsuoka, A.; Hachimori, A.; Sugawara, Y.; Shikama, K.; J. Biol. Chem., 1998, 273, 8607.

36. Perutz, M. F.; Heidner, E. J.; Ladner, J. E.; Beetlestone, J. G.; Ho, C.; Slade, E. F.; Biochemistry 1974, 13, 2187.

37. Ikeda-Saito, M.; Hori, H.; Andersson, L. A.; Prince, R. C.; Pickering, I. J.; George, G. N.; Sanders, C. R.; Lutz, R. S.; Mckelvey, E. J.; Mattera, R.; J. Biol. Chem. 1992, 267, 22843.

38. Smulevich, G.; Paoli, M.; De Sanctis, G.; Mantini, A.R.; Ascoli, F.; Coletta, M.; Biochemistry 1997, 36, 640.

39. Bujons, J.; Dikiy, A.; Ferrer, J.C.; Banci, L.; Mauk, A.G.; Eur. J. Biochem. 1997, 243, 72

40. Tada, T.; Watanabe, Y.; Matsuoka, A.; Ikeda-Saito, M.; Imai, K.; Ni-Hei, Y.; Shikama, K.; Biochim. Biophys. Acta 1998, 1387, 165.

41. Gilles-Gonzalez, M. A.; Gonzalez, G.; Perutz, M. F.; Biochemistry 1994, 33,8067 .

42. Kamimura, S.; Matsuoka, A.; Imai, K.; Shikama, K.; Eur. J. Biochem. 2003, 270,1424

43. Ilari, A.; Bonamore, A.; Farina, A; Johnson, K. A; Boffi, A.; J. Biol. Chem. 2002, 277, 23725 .

44. Bogumil, R.; Maurus, R.; Hildebrand, D. P.; Brayer, G. D., Mauk, G.; Biochemistry 1995, 34, 10483.

45. Suzuki, T.; Watanabe, Y.; Nagasawa, M.; Matsuoka, A.; Shikama, K.; Eur. J. Biochem. 2000, 267, 6166.

46. Morikis, D.; Champion, P. M.; Springer, B. A.; Egeberg, K. D.; Sligar, S. G.; J. Biol. Chem., 1990, 265, 12143.

47. Sigman, J. A.; Pond, A. E.; Dawson, J. H.; Lu, Y.; Biochemistry 1999, 38, 11122.

48. Boffi, A.; Das, T. K.; Longa, S. D.; Spagnuolo, C.; Rousseau, D. L.; Biophys. J. 1999, 77, 1143.

49. Smulevich, G.; Mauro, J. M.; Fishel, L. A.; English, A. M.; Kraut, J.; Spiro, T. G.; Biochemistry 1988, 27, 5477.

50. Vitello, L. B.; Erman, J. E.; Miller, M. A.; Mauro, J. M.; Kraut, J.; Biochemistry 1992, 31, 11524.

51. Ferrer, J. C.; Turano, P.; Banci, L.; Bertini, I.; Morris, I. K.; Smith, K. M.; Smith, M.; Mauk, A. G.; Biochemistry 1994, 33, 7819.

52. Bertini, I.; Gray, H.; Lippard, S. J.; Valentine, J. S.; Bioinorganic Chemistry, $1^{\text {st }}$ ed., University Science Books: Mill Valley, 1994.

53. Quilin, M. L.; Arduini, R. M.; Olson, J. S.; Phillips Jr, G. N.; J. Mol. Biol. 1993, 234, 140.

54. Rakshit, G.; Spiro, T. G.; Biochemistry 1974, 13, 5317.

55. Spiro, T. G.; Stong, J. D.; Stein, P.; J. Am. Chem. Soc. 1979, 101, 2648.

56. Yonetani, T.; Anni, H.; J. Biol. Chem. 1987, 262, 9547.

57. Spiro, T. G.; Burke, J. M.; J. Am. Chem. Soc. 1976, 98, 5482 